\title{
Conversion of DICOM Image in to JPEG, BMP and PNG Image Format
}

\author{
Nitin S. Ujgare \\ Assistant Professor, Maharashtra (INDIA)
}

\author{
Swati P. Baviskar \\ Assistant Professor, Maharashtra (INDIA)
}

\begin{abstract}
Digital Imaging and Communications in Medicine (DICOM) standard is an image archive system which allows itself to serve as an image manager that control the acquisition, retrieval, and distributions of medical images within entire picture archiving and communication systems (PACS).The DICOM technology is suitable when sending images between different departments within hospitals or/and other hospitals, and consultants. However, some hospitals lack the DICOM system.

In this paper proposed algorithm view and converts .dcm image files into bmp, png, jpeg standard image, whereby the image should be viewable, with common image viewing programs and its size should be small.
\end{abstract}

Keywords: PACS, Imaging standard, NEMA, Dataset, Metadata

\section{INTRODUCTION}

The necessity of telemedicine is widely increasing every day and online support is crucial to have faster diagnosis.. Computers are used not only to store or display images but also to make images or 3D models from the input series of data. Data are obtained from imaging devices that use complex methods, for example: CT, MRI, SPECT and PET. Digital Imaging and Communications in Medicine (DICOM) is a standard for handling, storing, Printing, and transmitting information in medical imaging. DICOM image file format stores the details about the image and the patient's details in the same file. DICOM differs from other data formats, that it groups information into data sets, which means that a file of a chest X-Ray image actually contains the patient ID within the file, so that the image can never be separated from this information by mistake The National Electrical Manufacturers Association (NEMA) holds the copyright to this standard. It was developed by the DICOM Standards committee [3].

\subsection{DCM File}

It is a binary file, which means that an ASCII-character-based text editor like Notepad does not show it properly. A DICOM file may be encoded in Little Endian or Big Endian byte orders. Elements in a DICOM file are always in ascending order, of tags. Private tags are always odd numbered.

\subsection{DICOM File Format}

A DICOM file consists of a header, followed by a pixel data. Each file contains a single DATA SET. A Data Set represents an instance of a real world Information Object. A DICOM Data Set does not include its total length. Data Set is constructed of data Elements.
(Data Elements contains the ENCODED VALUE of the attributes of that object).

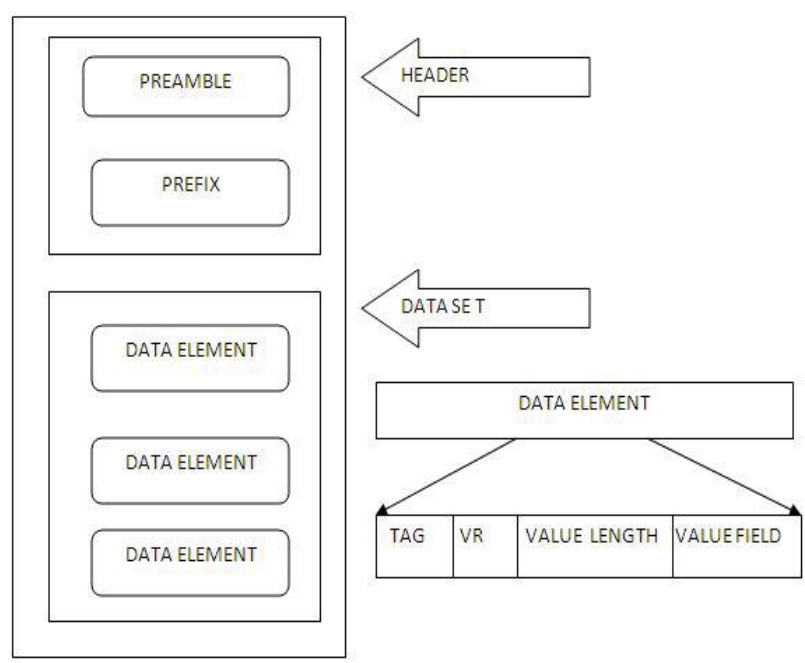

Fig. 1: DICOM file format

\subsubsection{Header}

The Header consists of "128" byte Preamble followed by a "4" byte DICOM Prefix. The header may or may not be included in the file. PREAMBLE 128 bytes=? PREFIX 4 bytes='D', 'I', 'C', 'M'. The Header comprises of Patient's name and other Patient particulars, and Image details. Important among image details are the Image dimensions i.e. Width, Height and Image bit per pixel. The DICOM standard does not require any structure for the fixed size preamble. It facilitates access to the images and other data in the DICOM file.

\subsubsection{Dataset}

Each file contains a single DATA SET. A Data Set represents an instance of a real world Information Object. A DICOM Data Set does not include its total length. Data Set is constructed of data Elements. (Data Elements contains the ENCODED VALUE of the attributes of that object).

\subsubsection{Data Element}

A Data Element is made up of fields. Common fields are: Data Element Tag, Value Length, and Value Field.

\subsection{Challenges}

DICOM image file format stores the details about the image and the patient's details in the same file. So size of images is very large, and that's why we are not able to send these images over the network for expert advice from the person who is situated in another country or state. The DICOM images require 
special type of viewer to view the image and it is not available everywhere.

\section{IMPLEMENTATION DETAILS}

Proposed method converts the .dcm file into generalized file format like jpeg, bmp, png etc. and also performs the following operation:

1. Extracting the patient details from the DICOM image.

2. Extracting the pixel data from the DICOM image.

3. Viewing the patient details and DICOM image.

4. Converting .dcm file into all possible file format.

5. Saving the patient details into text file.

\subsection{Dcm Image}

Input for this application is DICOM image which contain image and patient details together

\subsection{Read Image}

This block is used to read the image from specific directory. FileChooser class is used for selecting the image. After selection we get that image into the buffered area. Then input stream of the same image is created and set it as input to the decoding block.

\subsection{Decode}

This block is used to identify the various tags and also separate the various tags [2]. This block is mainly used for separating the tags of patient details and pixel information.

\subsubsection{Value representation}

The Value Representation of a Data Element describes the data type and format of that Data Element's [10].

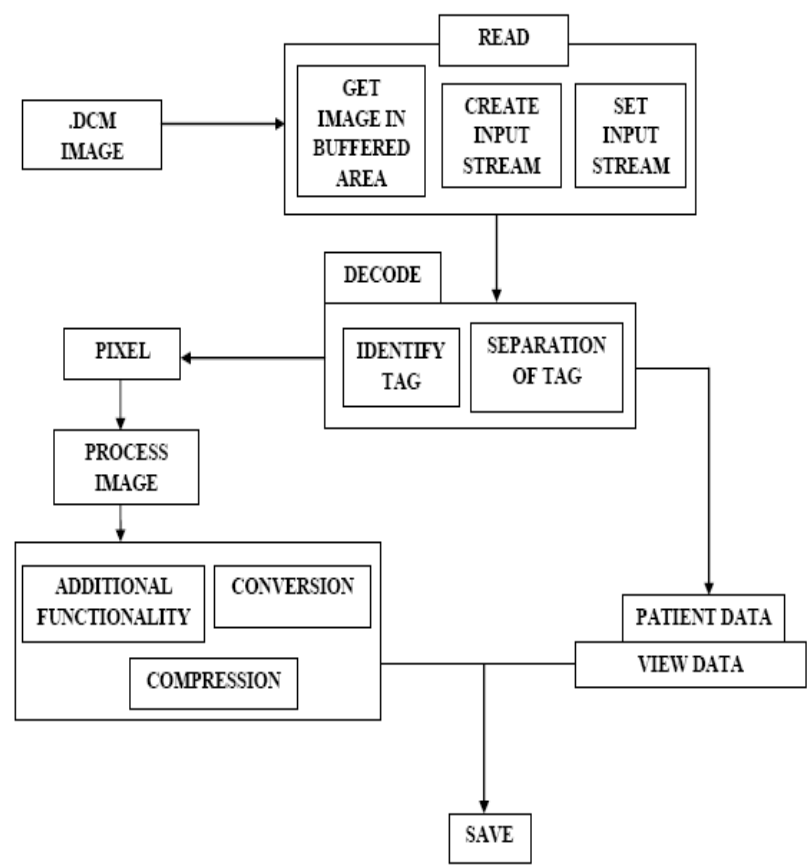

Fig. 2: System Architecture Diagram
Table 1. Value Representation

$\underline{\underline{2 \text { char }}} \underline{\underline{\text { code }}} \underline{\text { Max }} \quad \underline{\text { Remarks }}$

\begin{tabular}{|c|c|c|}
\hline $\mathrm{AE}$ & 16 & Application Entity/Name \\
\hline AS & 4 & Age String: nnnW or nnnM or nnnY \\
\hline AT & 4 & Attribute Tag gggg,eeee \\
\hline CS & 16 & Code String \\
\hline DA & 8 & $\begin{array}{l}\text { Date yyyymmdd (check for yyyy.mm.dd } \\
\text { also and convert) }\end{array}$ \\
\hline DS & 16 & $\begin{array}{l}\text { Decimal String may start with }+ \text { or }- \text { and } \\
\text { may be padded with } 1 \text { or } t \text { space }\end{array}$ \\
\hline DT & 26 & $\begin{array}{l}\text { Date Time } \\
\text { YYYYMMDDHHMMSS.FFFFFF\&ZZZ } \\
\text { (\&ZZZ is optional \& = + or }- \text { ) }\end{array}$ \\
\hline
\end{tabular}

FL $\quad 4 \quad$ Single precision floating pt number (float)

FD $16 \quad$ Double precision floating pt number (double)

IS $\quad 12 \quad$ Integer encoded as string. may be padded

LO $\quad 64 \quad$ Character string. can be padded. cannot contain \or any control chars except ESC

LT $10240 \quad$ Long Text. Leading spaces are significant. trailing spaces aren't

OB $\quad-\quad$ single trailing 0x00 to make even number of bytes. Transfer Syntax determines len

\begin{tabular}{|c|c|c|}
\hline OF & - & Other Float String. floats \\
\hline OW & - & Other Word String. words \\
\hline $\mathrm{PN}$ & - & $\begin{array}{l}\text { Person's Name 64byte max per component. } \\
5 \text { components. delimiter }=\wedge\end{array}$ \\
\hline SH & 16 & Short String. may be padded \\
\hline SL & 4 & signed long integer \\
\hline SQ & - & Sequence of zero or more items \\
\hline SS & 2 & signed short integer (word) \\
\hline ST & 1024 & Short Text of chars \\
\hline TM & 16 & $\begin{array}{l}\text { Time hhmmss.frac (or older format: } \\
\text { hh:mm:ss.frac) }\end{array}$ \\
\hline UI & 64 & $\begin{array}{l}\text { Unique Identifier (delimiter }=.) \text { 0-9 only, } \\
\text { trailing space to make even \# }\end{array}$ \\
\hline UL & 4 & Unsigned long integer \\
\hline UN & - & unknown \\
\hline US & 2 & Unsigned short integer (word) \\
\hline UT & - & Unlimited Text. trailing spaces ignored \\
\hline
\end{tabular}

A two-byte character string containing the VR of the Data Element. The VR for a given Data Element Tag shall be as defined by the Data Dictionary. The two character of VR shall 
be encoded using characters from the DICOM default character set.

\subsubsection{Value Multiplicity (VM)}

The Value Multiplicity of a Data Element specifies the number of Values that can be encoded in the Value Field of that data element. The VM of each Data Element is specified explicitly. If the number of Values that may be encoded in an element is variable, it shall be represented by two numbers separated by a dash e.g.

"1-10" means that there may be 1 to 10 Values in the element.

\subsubsection{The Data Set}

A Data Set represents an instance of a real world Information Object. A Data Set is constructed of Data Elements. Data Elements contain the encoded Values of Attributes of that object. The specific content and semantics of these Attributes are specified in Information Object Definitions. The construction, characteristics, and encoding of a Data Set and its Data Elements are discussed in this section. Pixel Data, Overlays, and Curves are Data Elements whose interpretation depends on other related elements.

\subsubsection{Data Elements}

A Data Element is uniquely identified by a Data Element Tag. The Data Elements in a Data Set shall be ordered by increasing Data Element Tag Number and shall occur at most once in a Data Set.

\begin{tabular}{|l|l|l|l|l|}
\hline $\begin{array}{l}\text { Data } \\
\text { Element }\end{array}$ & $\begin{array}{l}\text { Data } \\
\text { element }\end{array}$ & $\begin{array}{l}\text { Data } \\
\text { element }\end{array}$ & $\begin{array}{l}\text { Data } \\
\text { element }\end{array}$ & $\begin{array}{l}\text { Data } \\
\text { element }\end{array}$ \\
\hline Tag & VR & $\begin{array}{l}\text { Value } \\
\text { Length }\end{array}$ & $\begin{array}{l}\text { Value } \\
\text { Field }\end{array}$ \\
\hline
\end{tabular}

Fig. 3: Dataset

\section{Data Element Tag:}

An ordered pair of 16-bit unsigned integers representing the Group Number followed by Element Number.

\section{Value Length}

Either 16 or 32-bit (dependent on VR and whether VR is explicit or implicit) Unsigned integer containing the Explicit Length of the Value Field as the Number of bytes (even) that make up the Value. It does not include the length of the Data Element Tag, Value Representation, and Value Length Fields. A 32-bit Length Field set to Undefined Length (FFFFFFFFH). Undefined Lengths may be used for Data Elements having the Value Representation (VR) Sequence of Items (SQ) and Unknown (UN).

\section{Value Field}

An even number of bytes containing the Value(s) of the Data Element. The data type of Value(s) stored in this field is specified by the Data Element's VR. The VR for a given Data Element Tag can be determined using the Data Dictionary, or using the VR field if it is contained explicitly within the Data Element. The VR of Standard Data Elements shall agree with those specified in the Data Dictionary.

\subsection{Processing}

This block is used to process the DICOM image, in processing. Code is implemented for additional functionality and conversion of DICOM image to other generalized format.

\subsection{Proposed Algorithm}

Step 1: Select the DICOM file using read routine.

Step 2: Get the default parameter of file.

Step 3: Create the input stream of DICOM file.

Step 4: Set input stream using setinput method of reader class.

Step 5: Decode input stream.

Step 5.1: Read metadata of image.

Step 5.2: Read patient data from image.

Step 5.3: Read pixel information from the image.

Step 6: Display the DICOM image.

Step 7: Apply the various additional functionalities such as zoom in/out, rotate left/right etc.

Step 8: Display patient data.

Step 9: Convert the DICOM image into other image formats using write routine.

Step 10: Save the patient data into text file.

Step 11: Save the converted image.

The proposed algorithm works well for all the. $\mathrm{dcm}$ images of version 1.3.

\section{RESULTS}

We have implemented the proposed algorithm using JAVA NET beans IDE 7.0.1 on Windows 7 operating system. The average time required to convert the. $\mathrm{dcm}$ image into other format is one second. We have verified the proposed algorithm on dicom image BU001015MN-166-CL001v01_2005101414121985_2_5.dcm.

As shown in fig.4.1 DICOM image opened using DICOM image viewer software 


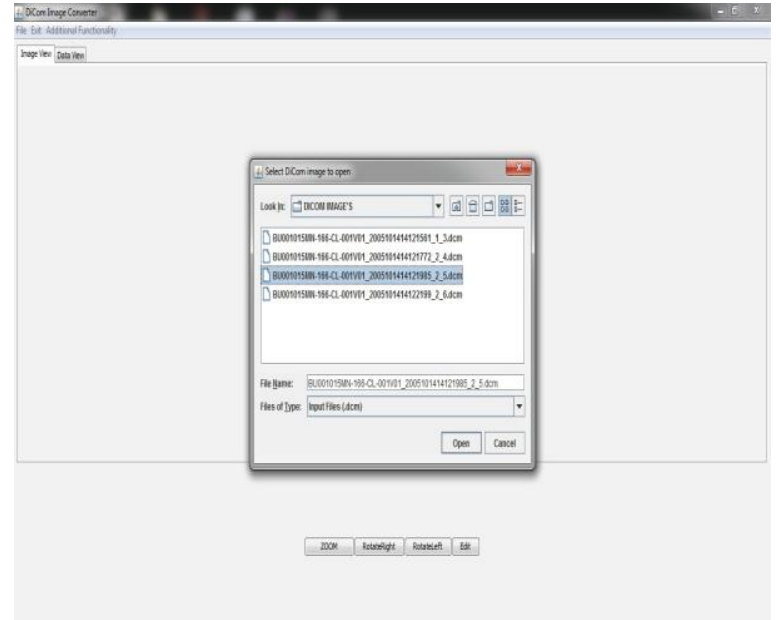

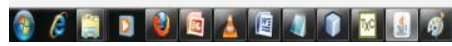

Fig. 4: Open DICOM Image

DICOM image view is shown in fig.5

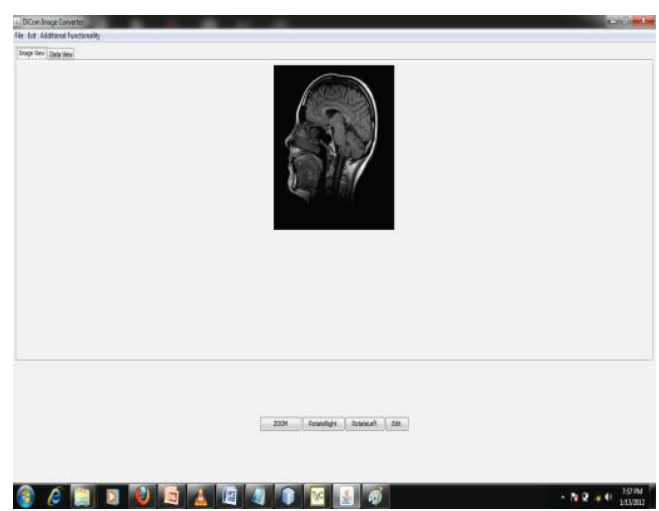

Fig. 5: View DICOM Image

User can view the data of patient which is stored with specified DICOM image as shown in fig. 6

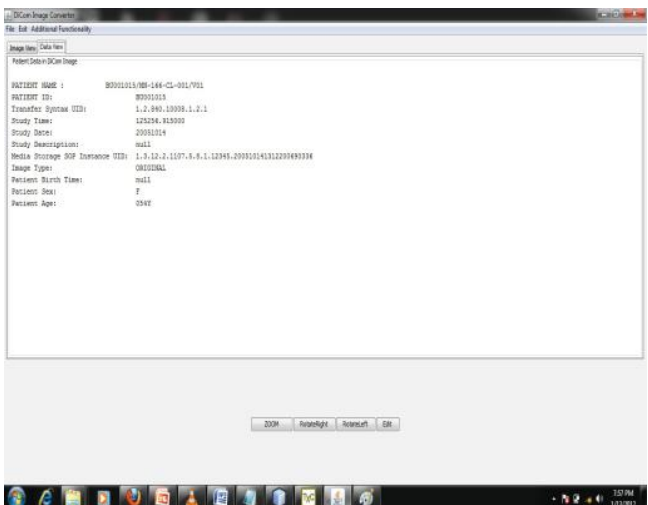

Fig. 6: View Patient Data

Following are the details of patient displayed in fig. 6
PATIENT NAME:

\section{PATIENT ID:}

Transfer Syntax UID

Study Time:

Study date:

Study Discription:

Media Storage SOP Instance UID

Image Type:

Patient Birth Time:

Patient Sex:

Patient Age:
BU001015/MN-166-CL-001/V01

BU001015

1.2.640.10008.1.2.1

125256.915000

20051014

null

1.3 .12 .2 .117 .5 .8 .1 .12345

Original

null

$\mathrm{F}$

054Y

For the better and clear view of the image, user can zoom in or zoom out the image as shown below in fig 7

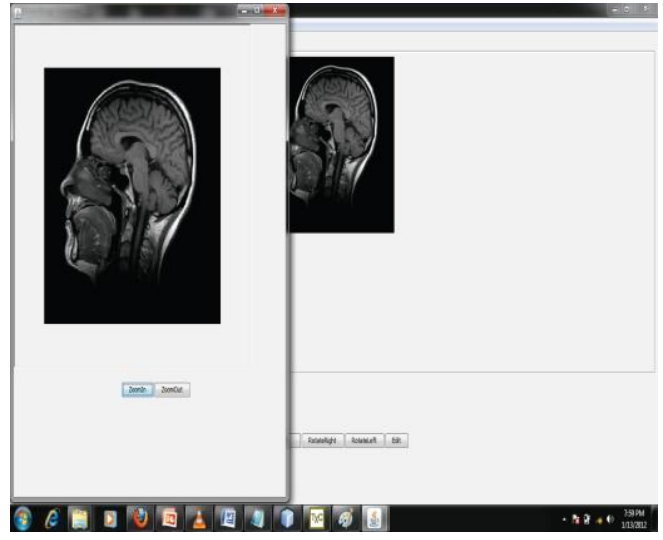

Fig. 7: Zoom In/Out

The image can be rotated to the left and right side as shown in fig. 8

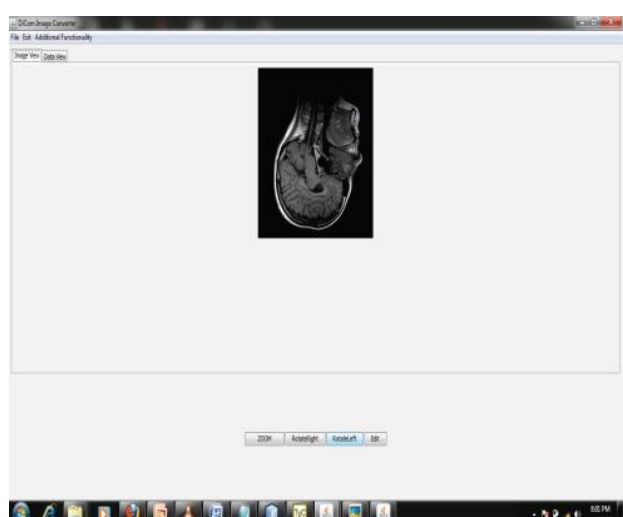

Fig. 8: Rotate Left/Right

In fig.9 DICOM image is converted in to other image formats An image is saved in universal formats (JPEG, bmp etc). 


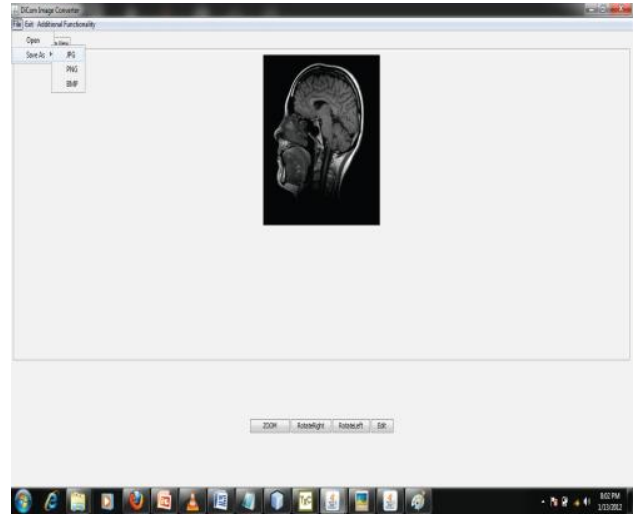

Fig. 9: Saving Images

\section{GRAPHICAL ANALYSIS}

The following graph represents the size of DICOM image and other universal image formats. As shown in figure.10

DICOM image requires the large space or memory to store the image while other universal formats like BMP, PNG \& JPEG requires less memory.

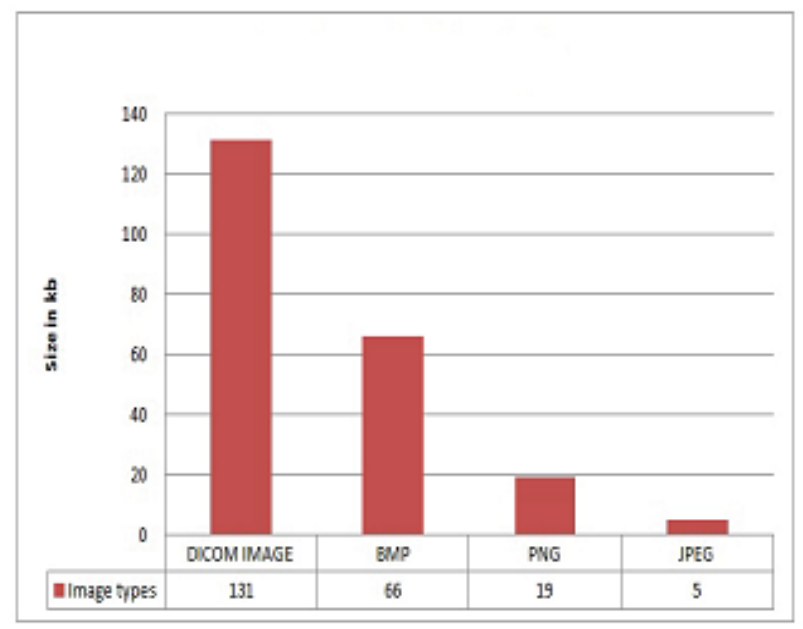

Fig.10: Comparison of DICOM image with BMP, PNG \& JPEG

The performance of a proposed algorithm can be evaluated visually and quantitatively based on application needs.

\section{CONCLUSION AND FUTURE WORK}

DICOM is one of the most ambitious medical image standards. It is developed to make image data standardized and easy to share between the equipment from different manufacturers. In this paper we have discussed how proposed algorithm is use to understand DICOM format and develop converter for the same. The initial Scope is to develop an image converter for DICOM images that can convert .dcm file into universal file format. It also consists of additional functionalities such as Zoom In/Out, Rotate Left/Right. Our future work focuses on developing the algorithm for conversion of DICOM image into other standard Image format. It is one of the best but not silver bullet method for conversion of .dcm image to all the other image format.

\section{REFERENCES}

[1] Kyucheol Cho, Jaejoon Kim, Se-Yoon Jung, Kyuhyeon Kim, Hyun-Kook Kuhng'Development of Medical Imaging Viewer Role in DICOM Standard", Daegu University, School of Computer and Communication Engineering **ETRI,Broadcasting Media Research Group, 0-7803-894091051, 2005 IEEE

[2] Wail A. Mousa, M. H. Shwehdi. and M. A. Abdul-Malek "Conversion of DICOM System Images To Common Standard Image Format Using Matlab", Asia SENSE. 2003 SENSOR. pg. $251-255$

[3] Mario Mustra, Kresimir Delac, Mislav Grgic "Overview of the DICOM Standard", 50th International Symposium ELMAR-2008, 10- 12 September 2008, Zadar, Croatia

[4] NEMA Publications,"DICOM strategic document", "Digital Imaging and Communications in Medicine (DICOM)" Ver. 8.0, April 2008

[5] Samit desai,Usha B.S."Medical image Transcoder for telemedicine based on wireless communication device",978-1-4244-8679-3/11/2011 IEEE

[6] Digital Imaging and Communications in Medicine (DICOM) Part 1: Introduction and Overview Published by National Electrical Manufacturers Association 1300 N. $17^{\text {th }}$ Street Rosslyn, Virginia 22209 USA

[7] Digital Imaging and Communications in Medicine (DICOM) Part 2: Conformance Published by National Electrical Manufacturers Association 1300 N. 17th Street Rosslyn, Virginia 22209 USA

[8] Digital Imaging and Communications in Medicine (DICOM) Part 3: Information Object Definitions Published by National Electrical Manufacturers Association $1300 \mathrm{~N}$. 17th Street Rosslyn, Virginia 22209 USA

[9] Digital Imaging and Communications in Medicine (DICOM) Part 4: Service Class Specifications Published by National Electrical Manufacturers Association 1300 N. $17^{\text {th }}$ Street Rosslyn, Virginia 22209 USA

[10] Digital Imaging and Communications in Medicine (DICOM) Part 5: Data Structures and Encoding Published by National Electrical Manufacturers Association $1300 \mathrm{~N}$. $17^{\text {th }}$ Street Rosslyn, Virginia 22209 USA 